\title{
Modified carbon-containing electrodes in stripping voltammetry of metals. Part II. Composite and microelectrodes
}

\author{
Natalya Yu. Stozhko - Natalya A. Malakhova • \\ Mikhail V. Fyodorov • Khiena Z. Brainina
}

Received: 19 October 2007 /Revised: 21 October 2007 / Accepted: 21 October 2007 /Published online: 21 December 2007

(C) The Author(s) 2007

\begin{abstract}
The second part of the review, which covers modified carbon-containing electrodes, describes composite and microelectrodes. Electrodes made of commercial and laboratory carbon-containing composite materials are discussed. Impregnated and thick-film electrodes and microelectrodes made of carbon fibers form a separate group. Various modifiers and methods of electrode modification are presented. Prospects for the future development of solid-state modified electrodes are considered.
\end{abstract}

Keywords Carbon-containing electrodes $\cdot$ Modified electrodes · Composite electrodes · Microelectrodes . Stripping voltammetry $\cdot$ Metal ions determination

\section{Graphitized carbon and composite electrodes}

The advancement of modern engineering and the use of new principles and approaches to the synthesis of carbon materials have facilitated the development of new commercial carbon-graphite materials with assigned properties. The use of the following commercially available electrode materials is reported in the literature: pencil lead [1-3]; spectral [4, 5], pyrolitic [6], and felt [7] graphites; carbon glass ceramic [8-10]; nonwoven graphite films [11]; carbon film resistors; and switches [12-16]. In most cases, electrodes of these materials are modified by metal films (mercury, copper, and bismuth), molecularly imprinted

\footnotetext{
N. Y. Stozhko $(\bowtie) \cdot$ N. A. Malakhova $\cdot$ M. V. Fyodorov $\cdot$

K. Z. Brainina

Ural State University of Economy,

8th March St. 62,

620219 Ekaterinburg, Russia

e-mail: sny@usue.ru
}

$\mathrm{TiO}_{2}$ [17]. The occurrence in the last decade of new types of carbon materials "from carbon nanotubes to edge plane pyrolytic graphite" $[18,19]$ has significantly changed the scope and sensitivity of electroanalytical methods for the measurement of diverse targets from metals ions to biological markers. Investigators considered in detail electrochemical characteristics [20] and practical use [21] of the "edge" plane pyrolytic graphite, which proved to have a wider interval of working potentials and a low detection limit as compared to those of the basal pyrolytic graphite and GC. For example, in situ bismuth film modified edge plane pyrolytic graphite electrode was successfully applied to the ultra trace simultaneous determination of cadmium(II) and lead(II) with detection limit $5.5 \cdot 10^{-10}$ and $4 \cdot 10^{-10} \mathrm{M}$, respectively [22].

Synthetic diamonds (nitrogen- $[23,24]$ and boron-doped [25-49]) have come into use quite recently for electrochemical measurements. In particular, a gold-coated, borondoped, diamond thin-film electrode was used for total inorganic arsenic detection in real water samples [50]. Unlike properties of other carbon materials, which are widely used in electroanalysis, properties of synthetic diamonds became the subject of comprehensive study just about 10 years ago. The research was hindered by two circumstances: shortage of the material and the absence of conduction. The situation radically changed with the advent of highly efficient methods for growing of polycrystalline diamond compounds.

A more efficient separation, accumulation, and determination of components is achieved with electrodes of composite materials made of graphite, carbon, glassy carbon, or diamond powders and binders such as paraffin, epoxy resins, methacrylate, silicon, styrene-acrylonitrile copolymer, polyester, and silica gel. The reviews [51-54] deal with properties and applications of various composite 
materials. Some original papers describe the use of unmodified electrodes made of graphite [55-62], glassy carbon [63], template carbon [64] -polymer composites, a composite based on natural and synthetic diamonds [65], nanocomposite made of carbon nanofibers [66], and a carbon-containing material prepared by a sol-gel technology [67, 68]. Much attention is given for the study of electrochemical properties and application of graphiteepoxy composite electrodes [69-72]. Specific features of the last material are its strength, chemical inertness, absence of impurities, nonswelling in water solutions, stability in organic solvents for prolonged periods, ease and simplicity of fabrication, and secure fixing of water-soluble reagents.

Table 1 gives examples of the use of modified composite electrodes in stripping voltammetry with the composite materials made under industrial and laboratory conditions using simple sol-gel, sono-gel, spin-casting, and molding technologies [73, 74-92]. The electrodes shown in the table can be divided into two groups: those made of carbon(soot, amorphous carbon) [73-79] and those made of graphite-containing [80-92] materials. It should be noted that the detection limit of graphite-containing materials is somewhat higher than that of carbon-containing materials. Composite electrodes may be used to achieve the electrochemical response of ground solids, iron pigments, and insoluble samples [93, 94].

The bulk or surface modification of composite materials by metals or organic compounds considerably reduces the detection limit for metal cations. Silicon-carbon electrodes were proposed for determination of $\mathrm{Cu}$ (II), $\mathrm{Pb}$ (II), and $\mathrm{Zn}$ (II) after modification by mercury [76]; Ni (II) after modification by dimethyl glyoxime [77]; Os (IV) after modification by 9-phenyl-3-fluorone [78]; and $\mathrm{Cu}$ (I), $\mathrm{Fe}$ (II), and Ni (II) after modification by neocupferron, phenanthroline derivatives, and dimethylglyoxime, respectively [79]. A satisfactory reproducibility of responses of elements to be determined, a high selectivity, and the time stability of sol-gel electrodes can be noted.

Modified electrodes of composite materials are attractive because not only their surface but also the bulk can be easily modified at the stage of the composite formation [73-75, 77-80, 84-86, 90-92], facilitating fabrication of more reproducible electrodes. Simplicity of technologies used for production of many current-conducting composite materials with preset properties makes them promising for electroanalysis applications.

\section{Impregnated graphite electrodes}

Impregnated graphite electrodes (IGE), which have found application in the electroanalytical practice, are basically of two types: those impregnated with a paraffin-polyethylene mixture and those with epoxy resins. IGE are widely used by electroanalysts in Russia. Unmodified [95, 96] and modified [97-129] IGE, shown in Table 2, are used in electrochemical inorganic analysis. IGE are modified by metals $(\mathrm{Hg}, \mathrm{Au}, \mathrm{Cu}, \mathrm{Tl}, \mathrm{Jr})$ [97, 98-114], polymer films fixing the modifier and capable of the ion exchange [115], and Mo (IV) oxocomplexes [116, 117]. The IGE surface has good adsorbability and, hence, is readily modified by reagents specific for determined ions [118-129]. The IGE surface is modified most frequently, but examples of the IGE bulk modification can be found too [118, 119]. In this case, porous graphite is first held in an acetone solution of 2-mercaptobenzoxazole and then impregnated with a twocomponent epoxy compound under exposure to ultrasound. IGE modified by this method were used to determine Ag (I) and $\mathrm{Hg}$ (II). They were additionally modified in situ by mercury and rhodamine for measurements of $\mathrm{Bi}$ (III) and $\mathrm{Au}$ (III), respectively.

IGE are widely used in abrasive stripping voltammetry developed by Scholtz et al. [130]. Abrasive stripping voltammetry is a new electroanalytical technique designed for qualitative and quantitative analysis of solid materials. The essence of this method is a recording of the voltammetric response of a set of solid microparticles of the analyte immobilized on an inert electrode (most frequently paraffin-impregnated graphite) both unmodified and modified, for example, with zeolites [131] immersed in an appropriate electrolyte. Voltammetry of microparticles is a rapidly growing field of research. This scheme was previously used to analyze metals, alloys, minerals, natural and synthetic sulfosalts, sulfides, and ceramic materials [132-138].

\section{Thick-film carbon-containing electrodes}

So-called "screen-printed" or "thick-film" electrodes have been used on a wide scale recently. They are reproducible and inexpensive. Thanks to their good electrochemical and metrological characteristics, these electrodes can serve for environmental monitoring and foodstuff quality control [139]. Thick-film electrodes are made of carbon- [140], graphite- [141, 142], carbon-nanotube-containing [143] inks or special current-conducting, e.g., graphite-epoxy, pastes. The design and the fabrication technology of the electrodes, as described, for example, in [144], allow easy modification of their surface by immobilizing a modifier on the electrode surface or adding it to the ink before the electrode is made.

Table 3 gives examples of the use of modified thick-film electrodes for voltammetric determination of elements [145, 146-192]. Metals and their oxides are the most common modifiers [145-163]. Sometimes the film electrodes are 


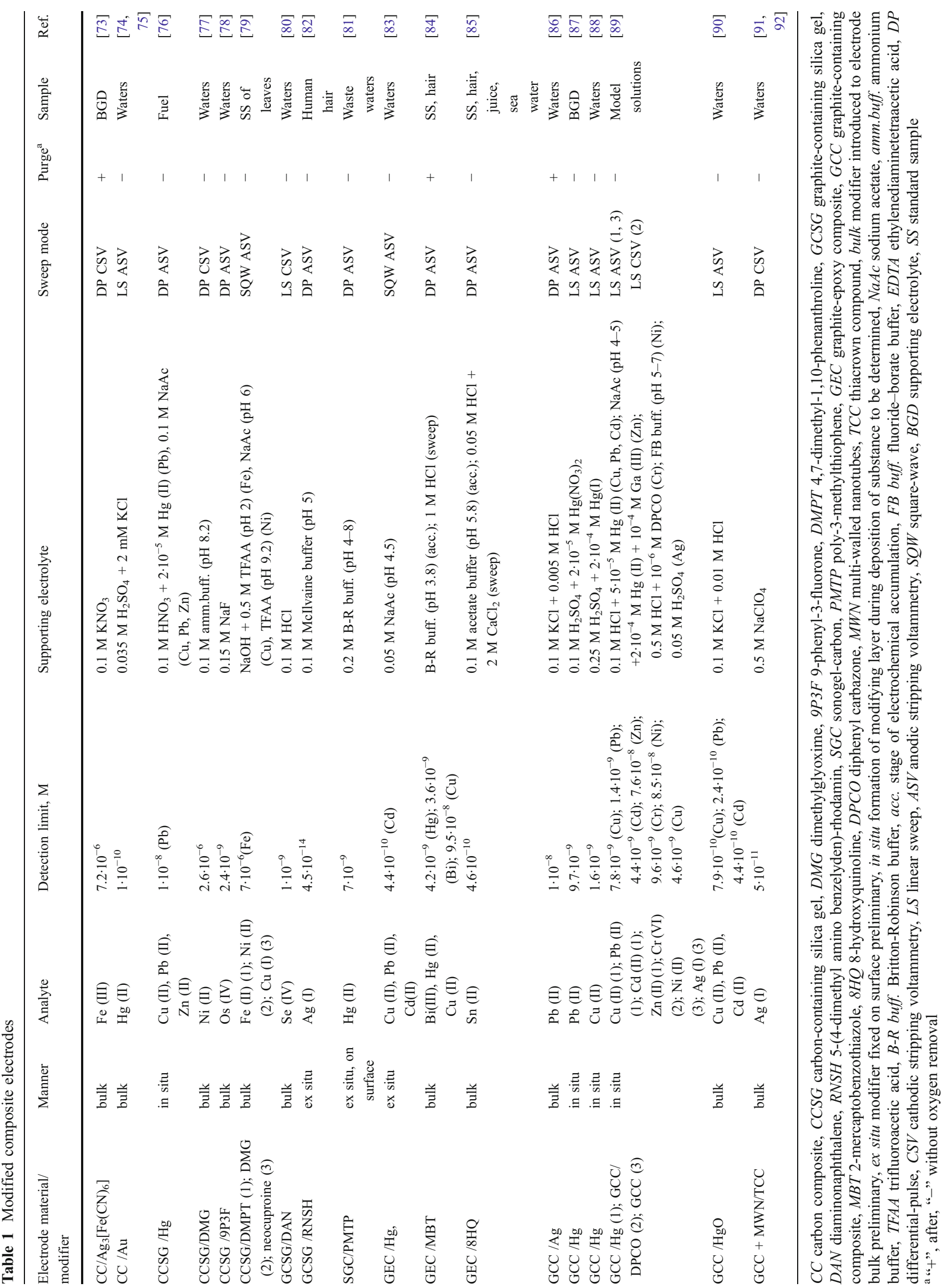


Table 2 Modified IGE

\begin{tabular}{|c|c|c|c|c|c|c|c|c|}
\hline $\begin{array}{l}\text { Electrode } \\
\text { material/ } \\
\text { modifier }\end{array}$ & Manner & Analyte & $\begin{array}{l}\text { Detection } \\
\text { limit, } \mathrm{M}\end{array}$ & Supporting electrolyte & $\begin{array}{l}\text { Sweep } \\
\text { mode }\end{array}$ & Purge & Sample & Ref. \\
\hline IGE-P /Hg & in situ & Pt (IV) & $1.3 \cdot 10^{-7}$ & $1 \mathrm{M} \mathrm{HCl}+13.4 \mathrm{mg} \mathrm{L}^{-1} \mathrm{Hg}$ (II) & LS ASV & - & Biomaterials & [97] \\
\hline IGE-P /Hg & in situ & $\begin{array}{l}\mathrm{Cu}(\mathrm{II}) \\
\mathrm{Pb}(\mathrm{II})\end{array}$ & $\begin{array}{l}3.7 \cdot 10^{-8}(\mathrm{~Pb}) \\
1.2 \cdot 10^{-7}(\mathrm{Cu})\end{array}$ & $\begin{array}{l}0.1 \mathrm{M} \mathrm{HCl}+1 \cdot 10^{-4} \mathrm{M} \mathrm{Hg} \text { (II) }+ \\
1.2 \mathrm{M} \mathrm{NaSCN}\end{array}$ & LS ASV & - & $\begin{array}{l}\text { Model } \\
\text { solutions }\end{array}$ & {$[98]$} \\
\hline $\begin{array}{l}\text { IGE-P / } \\
\mathrm{Hg}+\mathrm{Cu}\end{array}$ & in situ & Se (IV) & $2.5 \cdot 10^{-7}$ & $\begin{array}{l}0.1 \mathrm{M} \mathrm{HCl}+2.5 \cdot 10^{-5} \mathrm{M} \mathrm{Hg} \text { (II) }+ \\
3 \cdot 10^{-5} \mathrm{M} \mathrm{Cu}(\mathrm{II})\end{array}$ & SQW CSV & + & Foodstuffs & [99] \\
\hline IGE-R & ex situ & $\mathrm{Au}(\mathrm{III})$ & $5 \cdot 10^{-9}$ & $0.1 \mathrm{M} \mathrm{HCl}$ & LS ASV & - & Rocks & {$[100]$} \\
\hline IGE-R /Hg & ex situ & $\mathrm{Tl}$ (III) & $9.8 \cdot 10^{-12}$ & 0.05 M EDTA (pH 4.4) & DP ASV & + & Waters & {$[101]$} \\
\hline IGE-R /Hg & ex situ & $\begin{array}{c}\mathrm{Cu}(\mathrm{II}) \\
\mathrm{Pb}(\mathrm{II}) \\
\mathrm{Cd}(\mathrm{II})\end{array}$ & $\begin{array}{l}6.3 \cdot 10^{-9}(\mathrm{Cu}) \\
4.8 \cdot 10^{-10}(\mathrm{~Pb}) \\
8.9 \cdot 10^{-10}(\mathrm{Cd})\end{array}$ & $0.5 \mathrm{M} \mathrm{NaCl}(\mathrm{pH} 3)$ & LS ASV & - & $\begin{array}{r}\text { Natural } \\
\text { waters }\end{array}$ & [102] \\
\hline IGE-R /Hg & ex situ & $\begin{array}{l}\mathrm{Pb}(\mathrm{II}), \\
\mathrm{Cd}(\mathrm{II}) \\
\mathrm{Zn}(\mathrm{II})\end{array}$ & $10^{-10}$ & $0.02 \mathrm{M} \mathrm{KNO}_{3}+1 \mathrm{mM} \mathrm{HCl}(\mathrm{pH} 3)$ & LS ASV & - & $\begin{array}{l}\text { Model } \\
\text { solutions }\end{array}$ & [103] \\
\hline IGE-P /Au & ex situ & As (III) & $2.7 \cdot 10^{-10}$ & $\begin{array}{l}0.02-0.1 \mathrm{M} \text { EDTA + citrate } \mathrm{Na} \\
(\mathrm{pH} 4-5)\end{array}$ & LS ASV & + & $\begin{array}{l}\text { Drinking, } \\
\text { mineral } \\
\text { waters, } \\
\text { strong } \\
\text { drinks }\end{array}$ & [104] \\
\hline IGE-P /Au & ex situ & $\begin{array}{r}\text { As (III), } \\
\mathrm{Hg} \text { (II) }\end{array}$ & $\begin{array}{l}1.3 \cdot 10^{-8}(\mathrm{As}) \\
2.5 \cdot 10^{-9}(\mathrm{Hg})\end{array}$ & $0.1 \mathrm{M} \mathrm{HCl}$ & LS ASV & - & Natural waters & {$[105]$} \\
\hline IGE-P /Au & ex situ & $\mathrm{Se}(\mathrm{IV})$ & $3.8 \cdot 10^{-8}$ & $0.3 \mathrm{M} \mathrm{HClO}_{4}$ & LS ASV & - & Waters & {$[106]$} \\
\hline IGE-P /Au & $\begin{array}{l}\text { ex situ (1), } \\
\text { in situ (2) }\end{array}$ & $\begin{array}{l}\text { As (III) (1), } \\
\text { Se (IV) (1), } \\
\text { Hg (II) (2) }\end{array}$ & $\begin{array}{l}1.0 \mu \mathrm{g} \mathrm{g}^{-1}(\mathrm{As}) \\
0.6 \mu \mathrm{g} \mathrm{g}^{-1}(\mathrm{Se}) \\
0.3 \mu \mathrm{g} \mathrm{g}^{-1}(\mathrm{Hg})\end{array}$ & $\begin{array}{l}0.02-0.05 \mathrm{M} \text { EDTA (As); } 0.1-0.3 \mathrm{M} \\
\mathrm{HClO}_{4}(\mathrm{Se}) ; \mathrm{HClO}_{4}, \mathrm{HNO}_{3}, \mathrm{HCl}+ \\
\mathrm{Au}(\mathrm{III})(\mathrm{Hg})\end{array}$ & LS ASV & - & Toys & [107] \\
\hline IGE-P /Au & in situ & $\mathrm{Hg}$ (II) & $5 \cdot 10^{-10}$ & $\begin{array}{l}\left.0.02-0.05 \mathrm{M} \mathrm{HClO}_{4} \text { (or } 0.1 \mathrm{M} \mathrm{HNO}_{3}\right)+ \\
4 \cdot 10^{-6} \mathrm{M} \mathrm{Au}(\mathrm{III})+5 \mathrm{mM} \mathrm{Cl}^{-}\end{array}$ & LS ASV & - & $\begin{array}{l}\text { Natural } \\
\text { waters, } \\
\text { foodstuffs }\end{array}$ & $\begin{array}{l}{[108,} \\
109]\end{array}$ \\
\hline IGE-R /Au & ex situ & $\begin{array}{r}\text { As (III), } \\
\mathrm{Cu}(\mathrm{II})\end{array}$ & $\begin{array}{l}9.3 \cdot 10^{-8}(\mathrm{As}) \\
1.1 \cdot 10^{-7}(\mathrm{Cu})\end{array}$ & $0.01 \mathrm{M} \mathrm{H}_{3} \mathrm{PO}_{4}+1 \mathrm{mM}$ EDTA & LS ASV & - & $\begin{array}{l}\text { Model } \\
\text { solutions }\end{array}$ & {$[110]$} \\
\hline IGE-R /Au & ex situ & $\mathrm{Hg}$ (II) & $5 \cdot 10^{-10}$ & $0.1 \mathrm{M} \mathrm{HClO}_{4}+0.03 \mathrm{M} \mathrm{HCl}$ & DP ASV & + & Sea sediment & {$[111]$} \\
\hline IGE-R /Au & ex situ & $\mathrm{Hg}$ (II) & $8 \cdot 10^{-10}$ & $0.1 \mathrm{M} \mathrm{HCl}$ & LS ASV & - & $\begin{array}{l}\text { Waters, } \\
\text { foodstuffs }\end{array}$ & [112] \\
\hline $\begin{array}{l}\text { IGE-R /Au } \\
(1) ; \text { IGE-R/ } \\
\mathrm{Cu}(2)\end{array}$ & $\begin{array}{l}\text { ex situ (1), } \\
\text { in situ (2) }\end{array}$ & As (III) & $\begin{array}{r}2.3 \cdot 10^{-7}(1) \\
6.3 \cdot 10^{-7}(2)\end{array}$ & $\begin{array}{l}0.05 \mathrm{M} \text { EDTA (1); } 0.1 \mathrm{M} \mathrm{HCl}+ \\
5 \cdot 10^{-6} \mathrm{M} \mathrm{Cu} \text { (II) (2) }\end{array}$ & LS ASV & - & BGD & [113] \\
\hline $\begin{array}{l}\text { IGE-R/Tl or } \\
\text { In }\end{array}$ & in situ & $\mathrm{Hg}$ (II) & $1 \cdot 10^{-8}$ & $\begin{array}{l}0.1 \mathrm{M} \mathrm{HCl}+\mathrm{Tl}(\mathrm{I}) \text { or In (III) } \\
(10: 1=\mathrm{Hg}: \mathrm{Tl})\end{array}$ & LS ASV & - & $\begin{array}{l}\text { Model } \\
\text { solutions }\end{array}$ & [114] \\
\hline IGE-P/nafion & ex situ & $\operatorname{Ag}(\mathrm{I})$ & $2 \cdot 10^{-10}$ & $0.01 \mathrm{M} \mathrm{HNO}_{3}+0.03 \mathrm{M} \mathrm{NaCl}$ & LS ASV & - & Chemicals & {$[115]$} \\
\hline $\begin{array}{l}\text { IGE-P/Mo } \\
\text { (VI)-OC }\end{array}$ & in situ & As $(V)$ & $2.7 \cdot 10^{-9}$ & $\mathrm{NaCl}(\mathrm{pH} 2.5)+1.5 \cdot 10^{-3} \mathrm{M} \mathrm{Mo}(\mathrm{VI})$ & SQW CSV & + & $\begin{array}{l}\text { Waters, } \\
\text { foodstuffs }\end{array}$ & [116] \\
\hline $\begin{array}{l}\text { IGE-R or } \\
\text { IGE-P/Mo } \\
\text { (VI)-OC }\end{array}$ & in situ & As $(\mathrm{V})$ & $\begin{array}{l}3 \cdot 10^{-9}(\text { IGE-P); } \\
2 \cdot 10^{-10}(\text { IGE-R) }\end{array}$ & $\begin{array}{l}1.0 \mathrm{M}(\mathrm{Na}, \mathrm{H}) \mathrm{Cl}(\mathrm{pH} 2.5)+1.5 \cdot 10^{-3} \mathrm{M} \\
\mathrm{Mo}(\mathrm{VI})+30 \text { vol. } \% \text { 1,4-lioxane }\end{array}$ & DP CSV & - & $\begin{array}{l}\text { Natural, sea } \\
\text { waters, } \\
\text { foodstuffs }\end{array}$ & {$[117]$} \\
\hline $\begin{array}{l}\text { IGE-R/MBO/ } \\
\text { Rhodamine } \\
6 \mathrm{G}\end{array}$ & $\begin{array}{l}\text { ex situ/in } \\
\text { situ }\end{array}$ & $\mathrm{Au}(\mathrm{III})$ & $1.9 \cdot 10^{-10}$ & $\begin{array}{l}0.1 \mathrm{M} \mathrm{HCl}+1 \cdot 10^{-6} \mathrm{M} \text { Rhodamine } \\
6 \mathrm{G} \mathrm{(acc.)} ; 0.1 \mathrm{M} \mathrm{HCl} \text { (sweep) }\end{array}$ & $\begin{array}{l}\text { DP CVA } \\
\text { DP CSV }\end{array}$ & - & Sea water & [118] \\
\hline $\begin{array}{l}\text { IGE-R/MBO } \\
\text { (1); IGE-R/ } \\
\mathrm{MBO} / \mathrm{Hg} \\
(2)\end{array}$ & $\begin{array}{l}\text { ex situ (1), } \\
\text { ex situ/in } \\
\text { situ (2) }\end{array}$ & $\begin{array}{l}\mathrm{Ag}(\mathrm{I})(1), \\
\mathrm{Hg}(\mathrm{II})(1), \\
\mathrm{Bi}(\mathrm{III})(2)\end{array}$ & $\begin{array}{c}1.8 \cdot 10^{-10}(\mathrm{Ag}) \\
1.9 \cdot 10^{-9}(\mathrm{Hg}) \\
9.5 \cdot 10^{-9}(\mathrm{Bi})\end{array}$ & $\begin{array}{l}\text { Ag: } 0.1 \mathrm{M} \mathrm{NaAc}(\mathrm{pH} 4.3)(\text { acc.); } 0.07 \mathrm{M} \\
\mathrm{HCl} \text { (sweep); Hg: } 0.1 \mathrm{M} \mathrm{FB} \text { (pH 5.5) (acc.); } \\
0.075 \mathrm{M} \mathrm{KBr}+0.1 \mathrm{M} \mathrm{HNO}_{3} \text { (sweep); } \\
\text { Bi: B-R buff. (pH 3.8) }+5 \cdot 10^{-6} \mathrm{M} \mathrm{Hg} \text { (II) } \\
\text { (acc.); } 1.0 \mathrm{M} \mathrm{HCl} \text { (sweep) }\end{array}$ & DP ASV & + & $\begin{array}{l}\text { Sea water, } \\
\text { urine }\end{array}$ & [119] \\
\hline $\begin{array}{l}\text { IGE-R/ } \\
\text { DPCD }\end{array}$ & in situ & $\mathrm{Cr}(\mathrm{VI})$ & $1 \cdot 10^{-9}$ & $0.15 \mathrm{M} \mathrm{H}_{2} \mathrm{SO}_{4}+5 \cdot 10^{-6} \mathrm{M} \mathrm{DPCD}$ & LS CSV & - & $\begin{array}{l}\text { Natural, sea } \\
\text { waters, draw of } \\
\text { soils }\end{array}$ & $\begin{array}{r}{[120,} \\
121]\end{array}$ \\
\hline $\begin{array}{l}\text { IGE-R/ } \\
\text { DPCO }\end{array}$ & in situ & Mn (II) & $5 \cdot 10^{-9}$ & amm.buff. (pH 9.2) $+3 \cdot 10^{-5} \mathrm{M} \mathrm{DPCO}$ & LS CSV & - & $\begin{array}{l}\mathrm{SS} \text { of } \mathrm{Ni}- \\
\text { alloy, natural } \\
\text { waters }\end{array}$ & [122] \\
\hline
\end{tabular}


Table 2 (continued)

\begin{tabular}{|c|c|c|c|c|c|c|c|c|}
\hline $\begin{array}{l}\text { Electrode } \\
\text { material/ } \\
\text { modifier }\end{array}$ & Manner & Analyte & $\begin{array}{l}\text { Detection } \\
\text { limit, } \mathrm{M}\end{array}$ & Supporting electrolyte & $\begin{array}{l}\text { Sweep } \\
\text { mode }\end{array}$ & Purge & Sample & Ref. \\
\hline $\begin{array}{l}\text { IGE-P/ } \\
\text { ANTPR }\end{array}$ & in situ & Mo (VI) & $5 \cdot 10^{-10}$ & $\begin{array}{l}0.3-2.0 \mathrm{M} \mathrm{HCl}+0.05 \mathrm{M} \mathrm{ANTPR}+ \\
0.03 \mathrm{M} \mathrm{SCN}^{-}\end{array}$ & LS ASV & - & Natural waters & [123] \\
\hline $\begin{array}{c}\text { IGE-R / } \\
\text { DMG }\end{array}$ & in situ & Ni (II) & $3 \cdot 10^{-9}$ & $\begin{array}{l}0.2 \mathrm{M} \mathrm{NaCl}+2.5 \cdot 10^{-4} \mathrm{M} \mathrm{DMG}+ \\
0.03 \mathrm{M} \mathrm{NaCl}+\text { pyridine }\left(\mathrm{pH}^{8}-10\right)\end{array}$ & LS CSV & - & $\begin{array}{l}\text { Natural, waste } \\
\text { waters }\end{array}$ & {$[124]$} \\
\hline IGE-R /CE & $\begin{array}{r}\text { ex situ (1), } \\
\text { in situ (2) }\end{array}$ & Pd (II) & $\begin{array}{l}5 \cdot 10^{-9}(1) \\
1 \cdot 10^{-8}(2)\end{array}$ & $\begin{array}{l}0.1 \mathrm{M} \mathrm{HClO}_{4}(1) ; 0.1 \mathrm{M} \mathrm{HClO}_{4}+ \\
1 \cdot 10^{-2} \mathrm{gL}^{-1} \mathrm{CE}(2)\end{array}$ & LS ASV & - & BGD & $\begin{array}{r}{[125,} \\
126]\end{array}$ \\
\hline $\begin{array}{l}\text { IGE-P/ } \\
\text { ANTPR }\end{array}$ & in situ & $\mathrm{W}(\mathrm{VI})$ & $1 \cdot 10^{-9}$ & $\begin{array}{l}1 \mathrm{M} \mathrm{H}_{2} \mathrm{SO}_{4}+0.078 \mathrm{M} \mathrm{NH}_{4} \mathrm{SCN}+ \\
0.04 \mathrm{M} \mathrm{ANTPR}\end{array}$ & DP ASV & - & Waters & [127] \\
\hline $\begin{array}{l}\text { IGE-P / } \\
\text { 9P3F }\end{array}$ & in situ & $\begin{array}{l}\mathrm{Sb}(\mathrm{III}) \\
\mathrm{Sn}(\mathrm{IV})\end{array}$ & $1 \cdot 10^{-7}$ & $0.05 \mathrm{M} \mathrm{H}_{2} \mathrm{SO}_{4}+(2.5-3.0) \cdot 10^{-6} \mathrm{M} 9 \mathrm{P} 3 \mathrm{~F}$ & LS ASV & - & $\begin{array}{l}\mathrm{Ni}, \mathrm{Cr}, \mathrm{SS} \text { of } \\
\text { steels and } \\
\text { irons }\end{array}$ & $\begin{array}{r}{[128,} \\
129]\end{array}$ \\
\hline
\end{tabular}

For abbreviations aside from those defined here, see Table 1

$I G E-P$ impregnated graphite electrode soaked with paraffin and polyethylene, $I G E-R$ impregnated graphite electrode soaked with epoxy resin, $M o$ (VI)-OC Mo(VI) oxocomplex, $M B O$ mercaptobenzoxazole, DPCD diphenyl carbazide, ANTPR antipyrine, $C E$ crown ether, EDTA ethylenediaminetetraacetic acid, $F B$ phosphate buffer, $C V A$ cathodic voltammetry

modified by inorganic [164-167], metalorganic [168-174], and organic substances [175-180]; self-assembled layers [181-184]; macrocyclic compounds [185-187]; polymeric films [188, 189]; and ion-exchange resins [190, 191]. Modified thick-film electrodes provide the detection limit of elements at a level of $10^{-8}-10^{-10} \mathrm{M}$.

Plasma, laser, temperature, and electrochemical treatments of the surface of thick-film electrodes can influence its activity, increase the rate of electron transitions, and change the electrochemical process reversibility. Preliminary anodic polarization of an electrode [192] not only extended the range of the working potentials but also improved its sensitivity. Voltammetric, microscopic, and spectroscopic studies suggested that the reason for the favorable effect of the anodic activation on the electrochemical properties of the electrode was enrichment of its surface in new functional groups, removal of unwanted inclusions from the surface, and the increase in the surface roughness.

\section{Carbon microelectrodes}

Pioneering research into the kinetics of electrochemical reactions on microelectrodes (ME) with diameters of units to several tens of micrometers revealed that the electrodes demonstrated new properties as their diameter decreased. Specifically, capacitive currents, which are the main factor that limits fast electrochemical processes on solid electrodes, decrease to practically insignificant values, the mass transfer rate of the substance to ME increases, the electrochemical equilibrium is established much faster than on standard electrodes, and the ohmic potential drop iR is negligibly small for ME. The unique properties of ME make it possible to analyze and use solutions with a high ohmic resistance, including pure solvents, in voltammetry and extend the range of test compounds, primarily organic substances and extracts. Growing attention is given to ME because they can serve as the basis for development of simply designed and, hence, easily realizable miniature sensors and detectors for operation in flowing liquid systems and measurement of bioactive substances in vivo. The electrochemical properties, constructions, fabrication methods, and analytical capabilities of ME are described in reviews and original papers [193-199].

Nonmetal ME are made of carbon, graphitized and glassy-carbon fibers, filaments, or carbon pastes. Carbon fibers are synthesized by thermal decomposition of fibers based on cellulose or polyacrylonitrile. They have large chemical resistance and good current conduction. Along with favorable properties of the carbon fiber, one should take into account its capacity for hydrogen and oxygen chemisorption especially at a potential of $+1.4 \mathrm{~V}$, which can lead to irreversible changes of the surface and the decrease in the detection limit of elements. In this connection, it is recommended [200] to realize the electrochemical accumulation of elements on carbon-fiber ME at potentials when the oxygen and the hydrogen adsorption is at a minimum. Unlike metal ME available in a variety of shapes (hemispherical, conical, cylindrical, disk, as a ring, or as a disk with a ring), carbon ME are generally shaped as a disk. When ME are made of the 
Table 3 Modified planar carbon containing electrodes

\begin{tabular}{|c|c|c|c|c|c|c|c|c|}
\hline $\begin{array}{l}\text { Electrode } \\
\text { material }\end{array}$ & Modifier & Manner & Analyte & $\begin{array}{l}\text { Detection limit, } \\
\mathrm{M}\end{array}$ & Supporting electrolyte & $\begin{array}{l}\text { Sweep } \\
\text { mode }\end{array}$ & Sample & Ref. \\
\hline (C) ink & $\mathrm{Ag}$ & in situ & $\mathrm{Pb}$ (II) & $4.8 \cdot 10^{-9}$ & $\begin{array}{l}0.1 \mathrm{M} \mathrm{HNO}_{3}+50 \mathrm{mM} \\
\mathrm{NaCl}+500 \mu \mathrm{g} \mathrm{L}^{-1} \\
\mathrm{Ag}(\mathrm{I})\end{array}$ & DP ASV & BGD & {$[145]$} \\
\hline (C) ink & $\begin{array}{l}\mathrm{Ag} \text { nano or } \\
\mathrm{Au} \text { nano }\end{array}$ & ex situ & $\mathrm{Sb}$ (III) & $\begin{array}{l}6.8 \cdot 10^{-10} \\
(\mathrm{Ag} \text { nano }) \\
9.4 \cdot 10^{-10} \\
(\mathrm{Au} \text { nano })\end{array}$ & B-R buff. (pH 2) & DP ASV & $\begin{array}{l}\text { Sea } \\
\text { water, } \\
\text { PP }\end{array}$ & $\begin{array}{l}{[146,} \\
147]\end{array}$ \\
\hline (C) ink & $\mathrm{Au}$ & ex situ & $\mathrm{Hg}$ (II), $\mathrm{CH}_{3} \mathrm{Hg}^{+}$ & $2.5 \cdot 10^{-9}$ & $0.05 \mathrm{M} \mathrm{HCl}$ & SQW ASV & BGD & [148] \\
\hline (C) ink & $\mathrm{Bi}$ & ex situ & $\mathrm{Pb}$ (II) & $1.4 \cdot 10^{-9}$ & $0.01 \mathrm{M} \mathrm{NaAc}(\mathrm{pH} 4.5)$ & SQW ASV & Waters & [149] \\
\hline (C) ink & $\mathrm{Hg}$ & in situ & $\mathrm{Pb}$ (II) & $2.9 \cdot 10^{-9}$ & $\begin{array}{l}1.0 \mathrm{M} \mathrm{NaBr}+1.2 \mathrm{M} \mathrm{HCl}+ \\
50 \mu \mathrm{M} \mathrm{Hg}(\mathrm{II})+100 \mu \mathrm{g} \\
\mathrm{L}^{-1} \mathrm{In}(\mathrm{III})\end{array}$ & SQW ASV & Blood & {$[150]$} \\
\hline (C) ink & $\mathrm{Hg}$ & ex situ & $\mathrm{Pb}$ (II) & $1.4 \cdot 10^{-11}$ & $0.02 \mathrm{M} \mathrm{NaAc}(\mathrm{pH} 4.2)$ & $\begin{array}{l}\text { DP ASV } \\
\text { SQW ASV }\end{array}$ & $\begin{array}{l}\text { Waters, } \\
\text { urine }\end{array}$ & {$[151]$} \\
\hline (C) ink & $\mathrm{Hg}$ & in situ & $\begin{array}{l}\mathrm{Cu}(\mathrm{II}), \mathrm{Cd}(\mathrm{II}) \\
\mathrm{Pb}(\mathrm{II}), \mathrm{Zn}(\mathrm{II})\end{array}$ & $\begin{array}{r}1.9 \cdot 10^{-6}(\mathrm{Cu}) \\
6.3 \cdot 10^{-7}(\mathrm{Cd}) \\
3.1 \cdot 10^{-7}(\mathrm{~Pb}) \\
8.4 \cdot 10^{-7}(\mathrm{Zn})\end{array}$ & $\begin{array}{l}0.1 \mathrm{M} \mathrm{MgCl}_{2}+10 \mathrm{mM} \\
\mathrm{KHPh}+120 \mu \mathrm{g} \mathrm{L}^{-1} \\
\mathrm{Hg} \text { (II) }\end{array}$ & DP ASV & BGD & {$[152]$} \\
\hline (C) ink & $\mathrm{Hg}$ & ex situ & $\mathrm{Pb}$ (II), Cd (II) & $10^{-7}$ & $0.01 \mathrm{M} \mathrm{HCl}$ & SQW ASV & BGD & [153] \\
\hline (C) ink & $\mathrm{Hg}$ & ex situ & $\begin{array}{l}\mathrm{Cu}(\mathrm{II}), \mathrm{Pb}(\mathrm{II}), \\
\mathrm{Cd}(\mathrm{II})\end{array}$ & $\begin{array}{r}7.9 \cdot 10^{-10}(\mathrm{Cd}) \\
1.4 \cdot 10^{-10}(\mathrm{~Pb})\end{array}$ & $0.02 \mathrm{M} \mathrm{NaAc}(\mathrm{pH} 4.8)$ & DP ASV & Urine & {$[154]$} \\
\hline (C) ink & $\mathrm{Hg}$ & ex situ & $\begin{array}{l}\mathrm{Cu}(\mathrm{II}), \mathrm{Pb}(\mathrm{II}) \\
\mathrm{Cd}(\mathrm{II}), \mathrm{Zn}(\mathrm{II})\end{array}$ & $\begin{array}{c}1.6 \cdot 10^{-9}(\mathrm{Cu}) \\
8.9 \cdot 10^{-10}(\mathrm{Cd}) \\
4.8 \cdot 10^{-10}(\mathrm{~Pb}) \\
1.5 \cdot 10^{-9}(\mathrm{Zn})\end{array}$ & $0.1 \mathrm{M} \mathrm{NaAc}(\mathrm{pH} 4.0)$ & SQW ASV & $\begin{array}{l}\text { Waters, } \\
\text { wine }\end{array}$ & {$[155]$} \\
\hline (C) ink & $\mathrm{Hg} / \mathrm{Cu}$ & ex situ & $\mathrm{Pb}$ (II) & $3.9 \cdot 10^{-9}$ & $0.02 \mathrm{M} \mathrm{NaAc}$ & SQW ASV & Waters & {$[156]$} \\
\hline (G-E) paste & $\mathrm{Hg}$ & in situ & $\begin{array}{l}\mathrm{Cu}(\mathrm{II}), \mathrm{Pb}(\mathrm{II}) \\
\mathrm{Cd}(\mathrm{II})\end{array}$ & $\begin{array}{r}4.4 \cdot 10^{-9}(\mathrm{Cd}) \\
1.4 \cdot 10^{-9}(\mathrm{~Pb}) \\
1.6 \cdot 10^{-8}(\mathrm{Cu})\end{array}$ & $\begin{array}{l}0.1-0.5 \mathrm{M} \mathrm{HCl}+1 \cdot 10^{-4} \mathrm{M} \\
\mathrm{Hg} \text { (II) }\end{array}$ & $\begin{array}{l}\text { LS ASV, } \\
\text { DP ASV }\end{array}$ & $\begin{array}{l}\text { BGD, } \\
\text { blood }\end{array}$ & $\begin{array}{l}{[157,} \\
158]\end{array}$ \\
\hline (C) paste & $\mathrm{Hg}$ & in situ & $\begin{array}{l}\mathrm{Cd}(\mathrm{II}), \mathrm{Cu}(\mathrm{II}) \\
\mathrm{Pb}(\mathrm{II})\end{array}$ & $2.5 \cdot 10^{-8}(\mathrm{Cd})$ & $\begin{array}{l}0.1 \mathrm{M} \mathrm{KNO}_{3}+0.03 \mathrm{M} \mathrm{HNO}_{3}+ \\
1 \mathrm{mM} \mathrm{Hg}\left(\mathrm{NO}_{3}\right)_{2}+0.1 \mathrm{M} \\
\mathrm{Fe}\left(\mathrm{NO}_{3}\right)_{3}\end{array}$ & LS ASV & Waters & [159] \\
\hline $\begin{array}{l}\text { Carbonized } \\
\text { PAN }\end{array}$ & $\mathrm{Hg}$ & in situ & $\mathrm{Pb}$ (II); Cd (II) & $\begin{array}{l}3.1 \cdot 10^{-9}(\mathrm{~Pb}) \\
1.8 \cdot 10^{-9}(\mathrm{Cd})\end{array}$ & $\begin{array}{l}0.1 \mathrm{M} \mathrm{NaAc}(\mathrm{pH} 4.2)+10 \\
\mathrm{mg} \mathrm{L}^{-1} \mathrm{Hg}(\mathrm{II})\end{array}$ & SQW ASV & Blood & {$[160]$} \\
\hline (G) ink & $\mathrm{Hg}$ & ex situ & $\begin{array}{l}\mathrm{Cu}(\mathrm{II}), \mathrm{Pb}(\mathrm{II}) \\
\mathrm{Cd}(\mathrm{II})\end{array}$ & $\begin{array}{l}1.9 \cdot 10^{-9}(\mathrm{~Pb}) \\
8.9 \cdot 10^{-9}(\mathrm{Cd}) \\
7.9 \cdot 10^{-9}(\mathrm{Cu})\end{array}$ & $\begin{array}{l}0.1 \mathrm{M} \mathrm{HCl}(30 \mathrm{mM} \mathrm{NaAc}) \\
(\mathrm{pH} 4.7)\end{array}$ & SQW ASV & $\begin{array}{l}\text { Waters, } \\
\text { soil }\end{array}$ & $\begin{array}{l}{[161} \\
162]\end{array}$ \\
\hline $\begin{array}{l}\text { Soot }+ \\
\text { resin }+ \\
\text { PVC }\end{array}$ & $\mathrm{HgO}$ & bulk & $\begin{array}{l}\mathrm{Cu}(\mathrm{II}), \mathrm{Cd}(\mathrm{II}) \\
\mathrm{Pb} \text { (II) }\end{array}$ & $\begin{array}{l}1.6 \cdot 10^{-8}(\mathrm{Cu}) \\
8.9 \cdot 10^{-9}(\mathrm{Cd}) \\
4.8 \cdot 10^{-9}(\mathrm{~Pb})\end{array}$ & $\begin{array}{l}0.10 \mathrm{M} \mathrm{KNO}_{3}+0.024 \mathrm{M} \\
\mathrm{HCl}\end{array}$ & LS ASV & Waters & [163] \\
\hline (G) ink & $\mathrm{Hg}_{2} \mathrm{Cl}_{2}$ & ex situ & $\begin{array}{l}\mathrm{Cu}(\mathrm{II}), \mathrm{Pb}(\mathrm{II}), \\
\mathrm{Cd}(\mathrm{II}), \mathrm{Zn}(\mathrm{II})\end{array}$ & $\begin{array}{l}210^{-9}(\mathrm{Cu}) \\
2 \cdot 10^{-10}(\mathrm{Cd}) \\
1 \cdot 10^{-10}(\mathrm{~Pb}) \\
8 \cdot 10^{-10}(\mathrm{Zn})\end{array}$ & $\begin{array}{l}0.5 \mathrm{M} \mathrm{HCl}(\mathrm{Cu}, \mathrm{Pb}, \mathrm{Cd}) \\
\text { acetic-chloride buffer }(\mathrm{Zn})\end{array}$ & $\begin{array}{l}\text { LS ASV; } \\
\text { SQW ASV }\end{array}$ & $\begin{array}{c}\text { Waters, } \\
\text { wines }\end{array}$ & $\begin{array}{l}{[164,} \\
165]\end{array}$ \\
\hline (G-E) paste & $\begin{array}{l}\mathrm{Hg}_{2} \mathrm{Cl}_{2} / \\
\text { pyrocatechin }\end{array}$ & $\begin{array}{l}\text { ex situ/ } \\
\text { in situ }\end{array}$ & $\mathrm{Fe}(\mathrm{III})$ & $3.6 \cdot 10^{-10}$ & $\begin{array}{l}0.1 \mathrm{M} \mathrm{NaAc}+510^{-4} \mathrm{M} \\
\text { pyrocatechin }\end{array}$ & LS CSV & Waters & {$[166]$} \\
\hline (G) ink & $\mathrm{BiPO}_{4}$ & ex situ & $\begin{array}{l}\mathrm{Pb}(\mathrm{II}), \mathrm{Cd}(\mathrm{II}) \\
\mathrm{Zn}(\mathrm{II})\end{array}$ & $\begin{array}{l}2 \cdot 10^{-9}(\mathrm{~Pb}) \\
4 \cdot 10^{-9}(\mathrm{Cd}) \\
8 \cdot 10^{-9}(\mathrm{Zn})\end{array}$ & $0.1 \mathrm{M}$ acetate buffer $(\mathrm{pH} 4.5)$ & SQW ASV & BGD & {$[167]$} \\
\hline (G-E) paste & $\begin{array}{l}\text { Hg (1); } \\
\text { DPCD (2); } \\
\text { ANTPR (3); } \\
\text { DPCO (4); } \\
\text { DAN (5); } \\
\text { DMG(6) }\end{array}$ & in situ & $\begin{array}{l}\mathrm{Cu}(\mathrm{II}), \mathrm{Pb}(\mathrm{II}), \\
\mathrm{Cd}(\mathrm{II}), \mathrm{Zn} \text { (II) (1); } \\
\mathrm{Cr}(\mathrm{VI})(2) ; \\
\mathrm{Mo}(\mathrm{VI}) \\
\mathrm{W}(\mathrm{VI})(3) ; \\
\mathrm{Mn} \text { (II) (4); } \\
\mathrm{Se}(\mathrm{IV})(5) ; \\
\mathrm{Ni} \text { (II) (6) }\end{array}$ & $\begin{array}{l}10^{-9}(\mathrm{Cu}, \mathrm{Pb}, \mathrm{Cd} \\
\mathrm{Zn}, \mathrm{Cr}) ; 1 \cdot 10^{-9} \\
(\mathrm{Mo}) ; 3 \cdot 10^{-9} \\
(\mathrm{~W}) ; 6 \cdot 10^{-9} \\
(\mathrm{Mn}) ; 1 \cdot 10^{-8} \\
(\mathrm{Se}) ; 1 \cdot 10^{-9}(\mathrm{Ni})\end{array}$ & $\begin{array}{l}0.14 \mathrm{M} \mathrm{NaCl}+0.04 \mathrm{M} \mathrm{HCl}+ \\
1 \cdot 10^{-4} \mathrm{M} \mathrm{Hg}(\mathrm{II})(\mathrm{Cu}, \mathrm{Pb} \\
\mathrm{Cd}, \mathrm{Zn}) ; 0.15 \mathrm{M} \mathrm{H}_{2} \mathrm{SO}_{4}+ \\
5 \cdot 10^{-6} \mathrm{M} \mathrm{DPCD}(\mathrm{Cr}) ; 0.5- \\
0.9 \mathrm{M} \mathrm{HCl}+0.03-0.07 \mathrm{M} \\
\mathrm{NH}_{4} \mathrm{SCN}+0.02-0.05 \mathrm{M} \\
\text { ANTPR (Mo, W); amm.buff. } \\
+3 \cdot 10^{-5} \mathrm{M} \mathrm{DPCO}(\mathrm{Mn}) ; \\
0.1 \mathrm{M} \mathrm{HCl}+6.3 \cdot 10^{-5} \mathrm{M} \\
\mathrm{DAN}(\mathrm{Se}) ; 0.1 \mathrm{M} \mathrm{KCl}+ \\
2.5 \cdot 10^{-4} \mathrm{M} \mathrm{DMG}(\mathrm{Ni})\end{array}$ & $\begin{array}{l}\text { DP ASV } \\
(\mathrm{Cu}, \mathrm{Pb}, \\
\mathrm{Cd}, \mathrm{Zn}, \\
\mathrm{Mo}, \mathrm{W}) \\
\mathrm{LS} \mathrm{CSV} \\
(\mathrm{Cr}, \mathrm{Mn}, \\
\mathrm{Se}, \mathrm{Ni})\end{array}$ & Waters & $\begin{array}{l}{[168,} \\
169]\end{array}$ \\
\hline
\end{tabular}


Table 3 (continued)

\begin{tabular}{|c|c|c|c|c|c|c|c|c|}
\hline $\begin{array}{l}\text { Electrode } \\
\text { material }\end{array}$ & Modifier & Manner & Analyte & $\begin{array}{l}\text { Detection limit, } \\
\text { M }\end{array}$ & Supporting electrolyte & $\begin{array}{l}\text { Sweep } \\
\text { mode }\end{array}$ & Sample & Ref. \\
\hline (G-E) paste & $\begin{array}{l}\mathrm{Hg} \text {-DDC; } \\
\mathrm{Hg} \text {-PDTC } \\
\text { or } \\
\mathrm{HgAc}+ \\
\text { nafion (1); } \\
\mathrm{Au} \mathrm{PDTC} \text {, } \\
\mathrm{AuCl}_{3}+ \\
\text { nafion or } \\
\mathrm{HAuCl}_{4}(2)\end{array}$ & ex situ & $\begin{array}{l}\mathrm{Cu}(\mathrm{II}), \mathrm{Pb}(\mathrm{II}), \mathrm{Cd} \\
\text { (II), } \mathrm{Zn} \text { (II), Sn } \\
\text { (IV) (1); Hg (II) } \\
\text { (2); As(III) (2) }\end{array}$ & $\begin{array}{l}10^{-9}(\mathrm{Cu}, \mathrm{Pb}, \mathrm{Cd}, \\
\mathrm{Zn}) ; 8 \cdot 10^{-9} \\
(\mathrm{Sn}) ; \\
2.5 \cdot 10^{-11}(\mathrm{Hg}) \\
1.4 \cdot 10^{-8}(\mathrm{As})\end{array}$ & $\begin{array}{l}0.1 \mathrm{M} \mathrm{HCl}(\mathrm{Cu}, \mathrm{Pb}, \mathrm{Cd}, \mathrm{Zn}) \\
4.6 \mathrm{~g} \mathrm{~L}^{-1} \mathrm{NH}_{4} \mathrm{Cl}+9.6 \mathrm{~g} \mathrm{~L}^{-1} \\
\left(\mathrm{NH}_{4}\right)_{2} \mathrm{C}_{2} \mathrm{O}_{4}+9.4 \mathrm{~g} \mathrm{~L}^{-1} \\
\mathrm{HCl}+10 \mathrm{mgL}^{-1} \mathrm{MB}(\mathrm{Sn}) \\
0.1 \mathrm{M} \mathrm{H}_{2} \mathrm{SO}_{4}+4 \mathrm{mM} \mathrm{HCl} \\
(\mathrm{Hg}) ; 2 \mathrm{M} \mathrm{HCl}(\mathrm{As})\end{array}$ & $\begin{array}{l}\text { LS ASV; } \\
\text { DP ASV }\end{array}$ & $\begin{array}{l}\text { Juices, } \\
\text { waters }\end{array}$ & $\begin{array}{r}{[170-} \\
174]\end{array}$ \\
\hline (G-E) paste & NRS & in situ & Co (II) & $7.3 \cdot 10^{-12}$ & $\begin{array}{l}0.01 \mathrm{M} \text { citrate buffer }+5 \\
10^{-5} \mathrm{M} \mathrm{NRS}\end{array}$ & LS CSV & Waters & {$[175]$} \\
\hline (G) ink & SF & ex situ & $\begin{array}{l}\mathrm{Cu}(\mathrm{II}), \mathrm{Pb}(\mathrm{II}), \mathrm{Cd} \\
\text { (II), } \mathrm{Zn} \text { (II) }\end{array}$ & $\begin{array}{r}8 \cdot 10^{-9}(\mathrm{Cu}) \\
3 \cdot 10^{-9}(\mathrm{~Pb}) \\
2 \cdot 10^{-9}(\mathrm{Cd}) \\
8 \cdot 10^{-8}(\mathrm{Zn})\end{array}$ & $\begin{array}{l}0.1 \mathrm{M} \mathrm{NaAc}+0.35 \mathrm{M} \mathrm{NaCl}+ \\
10^{-3} \mathrm{M} \mathrm{HCl}\end{array}$ & LS ASV & Waters & [176] \\
\hline (G) ink & SF & bulk & Mn (II) & $1 \cdot 10^{-9}$ & $\begin{array}{l}0.1 \mathrm{M} \mathrm{NaCl}+0.1 \mathrm{M} \mathrm{amm} . \\
\text { buff. (pH 9.2) }\end{array}$ & LS CSV & Waters & {$[177]$} \\
\hline (C) ink & DMG & bulk & $\mathrm{Ni}$ (II) & $8.5 \cdot 10^{-8}$ & 0.1 M amm.buff. (pH 9.2) & DP CSV & Waters & [178] \\
\hline $\begin{array}{r}(\mathrm{SGG}) \\
\text { paste }\end{array}$ & DAN & ex situ & Se (IV) & $1.3 \cdot 10^{-9}$ & $0.1 \mathrm{M} \mathrm{HCl}$ & LS CSV & Waters & {$[179]$} \\
\hline (C) ink & Alizarin & bulk & $\mathrm{Al}$ (III) & $7.0 \cdot 10^{-7}$ & $0.1 \mathrm{M}$ amm.buff. (pH 9) & DP ASV & Soils & [180] \\
\hline (G) ink & $\begin{array}{l}\text { Sol-gel film } \\
\quad+\text { PT }\end{array}$ & ex situ & $\mathrm{Fe}(\mathrm{II}), \mathrm{Fe}(\mathrm{III})$ & $1 \cdot 10^{-6}$ & $0.1 \mathrm{M}$ acetate buffer & LS ASV & $\begin{array}{c}\text { Waters, } \\
\text { wines }\end{array}$ & $\begin{array}{l}{[181} \\
182]\end{array}$ \\
\hline (G) ink & $\begin{array}{l}\text { Ac-Phos } \\
\text { SAMMS }\end{array}$ & ex situ & $\mathrm{Pb}$ (II) & $4.4 \cdot 10^{-9}$ & $0.3-0.5 \mathrm{M} \mathrm{HCl}$ & SQW ASV & BGD & [183] \\
\hline (G) ink & Sal-SAMMS & ex situ & $\mathrm{Eu}(\mathrm{III})$ & $6.6 \cdot 10^{-8}$ & $\begin{array}{l}0.05 \mathrm{M} \text { acetate buffer } \\
(\mathrm{pH} 4.6-6.5)(\text { acc.); } \\
0.1-0.2 \mathrm{M} \mathrm{NH}_{4} \mathrm{Cl} \\
\text { (sweep) }\end{array}$ & SQW ASV & BGD & [184] \\
\hline (G) ink & $\begin{array}{l}\text { Calix }[4 \text { or } 6] \\
\text { arene }\end{array}$ & ex situ & $\mathrm{Pb}$ (II), Cd (II) & $\begin{array}{l}2.4 \cdot 10^{-8}(\mathrm{~Pb}) \\
2.5 \cdot 10^{-8}(\mathrm{Cd})\end{array}$ & $\begin{array}{l}1 \mathrm{M} \text { amm.buff. (pH 10.5-Pb, } \\
\mathrm{pH} \mathrm{6.5-Cd)} \mathrm{(acc.);0.1} \mathrm{M} \\
\mathrm{HCl} \text { (sweep) }\end{array}$ & DP ASV & Waters & $\begin{array}{l}{[185} \\
186]\end{array}$ \\
\hline (C) ink & CEBM & ex situ & $\mathrm{Pb}$ (II), Cd (II) & $\begin{array}{r}0.8 \cdot 10^{-8}(\mathrm{~Pb}) \\
0.6 \cdot 10^{-8}(\mathrm{Cd})\end{array}$ & $0.01 \mathrm{M} \mathrm{KNO}_{3}(\mathrm{pH} 2)$ & DP ASV & ES & {$[187]$} \\
\hline (G) ink & PAN & ex situ & $\mathrm{Pb}$ (II) & $7.2 \cdot 10^{-8}$ & $\begin{array}{c}0.37 \mathrm{M} \text { amm.buff. (pH 10.5) } \\
\text { (acc.); } 0.1 \mathrm{M} \mathrm{HCl} \text { (sweep) }\end{array}$ & DP ASV & Waters & {$[188]$} \\
\hline (C) ink & SPE/pcPVP & ex situ & $\mathrm{Hg}$ (II) & $1.6 \cdot 10^{-8}$ & $0.01 \mathrm{M} \mathrm{KCl}+0.02 \mathrm{M} \mathrm{H}_{2} \mathrm{SO}_{4}$ & DP ASV & $\mathrm{CP}$ & [189] \\
\hline (C) ink & $\begin{array}{l}\text { Semi chelate } \\
\text { Q10R }\end{array}$ & bulk & $\mathrm{Hg}$ (II) & $1.0 \cdot 10^{-11}$ & $\begin{array}{l}0.5 \mathrm{M} \mathrm{NaCl}+0.1 \mathrm{M} \mathrm{NaAc} \\
(\mathrm{pH} 4.5)\end{array}$ & DP ASV & Waters & [190] \\
\hline (G) ink & $\begin{array}{l}\text { Dowex 50W- } \\
\text { X8 }\end{array}$ & bulk & $\mathrm{Cu}$ (II) & $7.9 \cdot 10^{-9}$ & $5 \mathrm{mM} \mathrm{FB}(\mathrm{pH} \mathrm{5.7)}$ & DP AVA & Waters & [191] \\
\hline
\end{tabular}

For abbreviations aside from those defined here, see Tables 1 and 2

$(G)$ or $(C)$ ink graphite- or carbon-containing ink, (G-E) paste graphite-epoxy paste, $P A N$ polyacrylonitrile, $P V C$ polyvinylchloride, (SGG) paste sol-gel graphite paste, $D A N$ diaminonaphthalene, $N R S$ nitroso-R salt, $H g-D D C$ mercury diethyldithiocarbamate, $H g$ - $P D T C$ mercury pyrrolidine dithiocarbamate, $H g A c$ mercury(II) acetate, $A u$-PDTC aurum pyrrolidine dithiocarbamate, $A c-P h o s$ SAMMS acetamide phosphonic acid selfassembled monolayer on mesoporous silica, $S F$ hetaryl-substituted formazan, $S P E / p c P V P$ 1,5-dibromopentane partially (7\%) cross-linked poly(4vinylpyridine), $K H P h$ potassium hydrophthalate, $M B$ methylene blue, $P L H$ poly-L-histidine, $C E B M$ crown-ether based membrane, $A V A$ anodic voltammetry, $P P$ pharmaceutical preparations, $C P$ cosmetic productions, ES environmental samples

carbon fiber, the critical importance is attached to the technology used to cover the fiber with the insulating material because the insulation quality ultimately determines the mechanical strength and the operating stability of the electrode. A new technology by which ME are covered with a quartz film solves this problem to some extent. The film is deposited from the vapor phase including silicon carbide, hydrogen, and oxygen [201]. The components mutually interact to form quartz gradually precipitating on carbon fibers. Coatings made by this technology have no cracks and hermetically insulate the fibers.

The problem of measuring extremely low currents at the level of nano- and picoamperes on ME is solved by the use 
of up-to-date, highly sensitive electrochemical equipment or ME arrays (MEA) whose desired signal can be integrated and measured by standard electrochemical analyzers and polarographs. ME systems are made using methods of microelectronics, electron- and ion-beam technologies, Xray and high-temperature lithography, and photolithogra- phy. It should be noted that physical methods of producing ME systems are rather expensive and laborious.

The lower detection limit of determinable concentrations on electrodes made of a single unmodified carbon or graphite fiber is $10^{-7}-10^{-9} \mathrm{M}$ [202-212]. Still lower detection limits of $10^{-8}-10^{-10} \mathrm{M}$ were achieved with

Table 4 Modified carbon micro(nano)electrodes

\begin{tabular}{|c|c|c|c|c|c|c|c|c|}
\hline $\begin{array}{l}\text { Electrode } \\
\text { material/ } \\
\text { modifier }\end{array}$ & Manner & Analyte & $\begin{array}{l}\text { Detection } \\
\text { limit, } \mathrm{M}\end{array}$ & Supporting electrolyte & Sweep mode & Purge & Sample & Ref. \\
\hline $\begin{array}{l}\mathrm{GF}(\mathrm{d}= \\
8 \mu \mathrm{m}) / \mathrm{Hg}\end{array}$ & in situ & $\mathrm{Cd}(\mathrm{II}), \mathrm{Pb}(\mathrm{II})$ & $5 \cdot 10^{-10}$ & $0.84 \mathrm{mM} \mathrm{NaAc}$ & SQW ASV & - & BGD & {$[213]$} \\
\hline $\mathrm{CF} / \mathrm{Hg}$ & in situ & $\mathrm{Pb}(\mathrm{II})$ & $4.8 \cdot 10^{-10}$ & $1 \cdot 10^{-7} \mathrm{M} \mathrm{Hg}$ (II) & LS ASV & - & $\begin{array}{r}\text { Natural } \\
\text { waters }\end{array}$ & {$[214]$} \\
\hline $\begin{array}{l}\mathrm{CF}(\mathrm{d}= \\
8 \mu \mathrm{m}) / \mathrm{Hg}\end{array}$ & in situ & $\mathrm{Zn}(\mathrm{II})$ & $1 \cdot 10^{-7}$ & $0.15 \mathrm{M} \mathrm{FB}+\mathrm{Hg}\left(\mathrm{NO}_{3}\right)_{2}$ & SQW ASV & - & Insulin & {$[215]$} \\
\hline $\mathrm{CF} / \mathrm{Hg}$ & in situ & $\mathrm{Cu}(\mathrm{II}), \mathrm{Pb}(\mathrm{II})$ & $\begin{array}{l}2.9 \cdot 10^{-9} \\
(\mathrm{~Pb}) \\
3.1 \cdot 10^{-8} \\
(\mathrm{Cu})\end{array}$ & $\begin{array}{l}0.1 \mathrm{M} \mathrm{KNO}_{3}+0.1 \mathrm{M} \mathrm{HNO}_{3} \\
(\mathrm{pH} 1)+0.01 \mathrm{mM} \mathrm{Hg}\left(\mathrm{NO}_{3}\right)_{2}\end{array}$ & LS ASV & + & $\begin{array}{l}\text { Natural } \\
\text { and } \\
\text { mineral } \\
\text { waters }\end{array}$ & {$[216]$} \\
\hline $\begin{array}{l}\mathrm{CF}(\mathrm{d}= \\
30 \mu \mathrm{m}) / \mathrm{Hg}\end{array}$ & in situ & $\begin{array}{l}\mathrm{Pb}(\mathrm{II}), \mathrm{Cd}(\mathrm{II}) \\
\mathrm{Zn} \text { (II) }\end{array}$ & $\begin{array}{c}1 \cdot 10^{-10} \\
(\mathrm{Zn})\end{array}$ & $\begin{array}{l}0.01 \mathrm{M} \mathrm{KCl}+1 \cdot 10^{-6} \mathrm{M} \\
\mathrm{Hg}(\mathrm{II})+0.033 \mathrm{M} \mathrm{HNO}_{3}\end{array}$ & LS ASV & + & $\begin{array}{l}\text { Natural } \\
\text { waters, } \\
\text { rainfalls }\end{array}$ & {$[217]$} \\
\hline $\begin{array}{l}\mathrm{CF}(\mathrm{d}= \\
6 \mu \mathrm{m}) / \mathrm{Hg}\end{array}$ & ex situ & $\mathrm{Cu}(\mathrm{II}), \mathrm{Pb}(\mathrm{II})$ & $1 \cdot 10^{-7}$ & $0.1 \mathrm{M} \mathrm{NaAc}$ & LS ASV & + & $\begin{array}{r}\text { Natural } \\
\text { waters }\end{array}$ & {$[218]$} \\
\hline $\begin{array}{l}\mathrm{GCF}(\mathrm{d}= \\
10 \mu \mathrm{m}) / \mathrm{Hg}\end{array}$ & ex situ & $\mathrm{Zn}$ (II) & $7.6 \cdot 10^{-7}$ & $10 \mathrm{mM} \mathrm{KNO}_{3}+10 \mathrm{mM} \mathrm{HNO}_{3}$ & SQW ASV & - & BGD & [219] \\
\hline $\begin{array}{l}\mathrm{GCF}(\mathrm{d}= \\
8 \mu \mathrm{m}) / \mathrm{Hg}\end{array}$ & ex situ & $\begin{array}{l}\mathrm{Cu}(\mathrm{II}) ; \mathrm{Pb}(\mathrm{II}) \\
\mathrm{Cd}(\mathrm{II})\end{array}$ & $10^{-8}$ & $0.01 \mathrm{M} \mathrm{HCl}$ & $\begin{array}{l}\text { DP ASV, } \\
\text { SQW ASV }\end{array}$ & + & $\begin{array}{r}\text { Natural } \\
\text { waters }\end{array}$ & {$[220]$} \\
\hline $\begin{array}{l}\mathrm{CF}(\mathrm{d}= \\
30 \mu \mathrm{m}) / \mathrm{Au}\end{array}$ & in situ & $\mathrm{Hg}$ (II) & $8.1 \cdot 10^{-11}$ & $\begin{array}{l}0.1 \mathrm{M} \mathrm{HClO}_{4}+5 \mathrm{mM} \mathrm{HCl}+ \\
5 \cdot 10^{-7} \mathrm{M} \mathrm{Au} \text { (III) }\end{array}$ & DP ASV & - & $\begin{array}{r}\text { Natural } \\
\text { waters }\end{array}$ & {$[221]$} \\
\hline $\mathrm{CF} / \mathrm{Bi}$ & in situ & $\begin{array}{l}\mathrm{Tl}(\mathrm{I}), \mathrm{Cd}(\mathrm{II}) \\
\mathrm{Zn} \text { (II), } \mathrm{Pb} \text { (II) }\end{array}$ & $10^{-8}-10^{-9}$ & $\begin{array}{l}0.1 \mathrm{M} \mathrm{NaAc}^{(\mathrm{pH} 4.5)}+400 \\
\mu \mathrm{g} \mathrm{L}^{-1} \mathrm{Bi} \text { (III) }\end{array}$ & SQW ASV & - & BGD & {$[222]$} \\
\hline $\begin{array}{l}\mathrm{CF} / \mathrm{Bi} / \\
\text { cupferron }\end{array}$ & $\begin{array}{l}\text { ex situ/ in } \\
\text { situ }\end{array}$ & U (VI) & $1.3 \cdot 10^{-9}$ & $\begin{array}{l}0.1 \mathrm{M} \mathrm{NaAc}(\mathrm{pH} 4.6)+ \\
7.5 \cdot 10^{-5} \mathrm{M} \text { cupferron }\end{array}$ & SQW ASV & - & $\begin{array}{l}\text { Sea } \\
\text { waters }\end{array}$ & {$[223]$} \\
\hline $\begin{array}{l}\mathrm{CF}(\mathrm{d}= \\
7 \mu \mathrm{m}) / \mathrm{Bi} \\
(1) ; \mathrm{CF} / \mathrm{Bi} / \\
\mathrm{DMG}(2)\end{array}$ & $\begin{array}{l}\text { ex situ } \\
(1), \text { ex } \\
\text { situ/in } \\
\text { situ (2) }\end{array}$ & $\begin{array}{l}\mathrm{Pb}(\mathrm{II})(1), \mathrm{Cd} \\
\text { (II) (1), Co (II) } \\
\text { (2), Ni (II) (2) }\end{array}$ & $\begin{array}{l}1.2 \cdot 10^{-9} \\
(\mathrm{Co}) \\
1.5 \cdot 10^{-9} \\
(\mathrm{Ni})\end{array}$ & $\begin{array}{l}0.05 \mathrm{M} \mathrm{NaAc}(\mathrm{pH} 4.5)(\mathrm{Cd} \\
\mathrm{Pb}) ; 0.01 \mathrm{M} \text { amm.buff. } \\
(\mathrm{pH} 9.2)+5 \cdot 10^{-5} \mathrm{M} \mathrm{DMG} \\
(\mathrm{Co}, \mathrm{Ni})\end{array}$ & $\begin{array}{l}\text { SQW CSV } \\
(\mathrm{Co}, \mathrm{Ni}) \\
\text { SQW ASV } \\
(\mathrm{Cd}, \mathrm{Pb})\end{array}$ & - & $\begin{array}{l}\text { Model } \\
\text { solutions }\end{array}$ & {$[224]$} \\
\hline CMEA /Hg & in situ & $\mathrm{Cd}(\mathrm{II}), \mathrm{Pb}(\mathrm{II})$ & $\begin{array}{l}1.4 \cdot 10^{-9} \\
(\mathrm{~Pb}) \\
2.7 \cdot 10^{-9} \\
(\mathrm{Cd})\end{array}$ & $\begin{array}{l}0.1 \mathrm{M} \mathrm{NaAc}+5 \cdot 10^{-5} \mathrm{M} \\
\mathrm{Hg}\left(\mathrm{NO}_{3}\right)_{2}\end{array}$ & SQW ASV & + & $\begin{array}{l}\text { Drinking } \\
\text { water }\end{array}$ & {$[225]$} \\
\hline $\begin{array}{l}\text { CMEA }(\mathrm{d}= \\
7 \mu \mathrm{m}) / \mathrm{Hg}\end{array}$ & in situ & $\mathrm{Cd}(\mathrm{II}), \mathrm{Pb}(\mathrm{II})$ & $1 \cdot 10^{-6}$ & $\begin{array}{l}0.1 \mathrm{M} \mathrm{KNO}_{3}(\mathrm{pH} 1.7 \\
\left.\mathrm{HNO}_{3}\right)+0.38 \mathrm{mM} \mathrm{Hg} \text { (II) }\end{array}$ & LS ASV & + & $\begin{array}{l}\text { Model } \\
\text { solutions }\end{array}$ & {$[226]$} \\
\hline $\begin{array}{l}\text { CMEA }(\mathrm{d}= \\
10 \mu \mathrm{m}) / \mathrm{Hg}\end{array}$ & in situ & $\mathrm{Pb}$ (II) & $1.3 \cdot 10^{-7}$ & $\begin{array}{l}1.2 \mathrm{M} \mathrm{HCl}+0.046 \mathrm{mM} \\
\mathrm{Hg} \text { (II) }\end{array}$ & SQW ASV & - & Blood & [227] \\
\hline CNTA/Bi & in situ & $\mathrm{Pb}$ (II), Cd (II) & $\begin{array}{l}2 \cdot 10^{-10} \\
(\mathrm{~Pb}) \\
3.6 \cdot 10^{-10} \\
(\mathrm{Cd})\end{array}$ & $\begin{array}{l}0.1 \mathrm{M} \text { acetate buffer }(\mathrm{pH} 4.5)+ \\
5 \cdot 10^{-4} \mathrm{\mu gL}^{-1} \mathrm{Bi}(\mathrm{III})\end{array}$ & SQW ASV & - & BGD & [228] \\
\hline
\end{tabular}

For abbreviations aside from those defined here, see Tables 1 to 3

$G F$ graphite fiber, $C F$ carbon fiber, $G C F$ glassy-carbon fiber, $C F A$ carbon fiber array, $C M E A$ carbon microelectrode array, $C N T A$ carbon nanotubes array 
mercury-, gold-, and bismuth-modified ME, MEA and CNTA [213-227]; see Table 4.

\section{Future trends}

Having passed the point of centuries and marched into the twenty-first century, voltammetry is advancing in the direction concisely characterized by Wang [229] as "faster, cheaper, simpler and better." This is largely because the main line of research changed from elaboration of the theory and equipment to development and application of new electrodes, sensors, and transducers in electroanalysis. Special emphasis is on the solution/electrode interface and the selective response formed on this interface. A new generation of electrodes with chemical or physical modification of the surface is under development. A great number of studies concerned with selection of modifiers, their immobilization on the solid surface, and the use of chemically modified electrodes (ChME) provide strong evidence for sincere interest paid by researchers to this problem. A wealth of experience has been accumulated in development of ChME, some features specific to fixing of modifiers on various matrices have been established, and conditions have been formulated for the electrochemical accumulation and determination of some substances on modified electrodes. However, the phenomenological stage of the ChME development is not complete because a diversity of modifiers and variants of their immobilization on electrodes will give researchers a wide scope of work for a long time to come.

It follows from the literature survey that a very promising and hopeful line of development of electrochemical sensors is the use of nanoparticles in electroanalysis [230-232] and the creation of micro- and nanoelectrode arrays, which can uniquely measure the electrochemical response in nonconductive media and unstirred electrolytes. However, the technologies used for the production of ME ensembles are extremely complicated and unavailable for ordinary research laboratories, suggesting the need to develop new methods of their fabrication. The creation of ordered nanostructures by traditional methods is an impracticable task, and in this case, it is appropriate to use the "bottom to top" rather than the "top to bottom" strategy. A proven fact is that nanoparticles of similar dimensions, which are synthesized "from top" by dispersion and "from bottom" by construction from atoms, are sharply different. The structure of the initial sample is preserved in dispersion particles, whereas particles formed by aggregation of atoms can have another arrangement of atoms influencing the electronic structure and chemical properties. Furthermore, processes of self-organization and evolution of modified layers on graphite-containing electrodes are quite possible because of the high surface energy of the graphitecontaining matrix.

It is reasonable to expect that the next stage of development of solid-state electrodes will include comprehensive studies of the electrode surface, elaboration of physical concepts for prediction of properties and characteristics of modified electrodes, and transition from micro- to ultramicro- and nanostructures on the electrode surface that would profoundly alter its properties. At this stage of development, study, and use of electrochemical sensors, we shall have to answer a number of questions: how the surface microgeometry influences the electrochemical activity of the modifier and the response parameters, how machining of the surface influences the electrochemical activity of the modifier and the electrode process, how the surface of the modified electrode can be made reproducible and the response stable, how to avoid formation of passivating films which impair electroanalytical performance of the electrodes, and other questions. This task will require performing a comprehensive systematic study of all links in the integral chain "electrode surface-modifier-response-SV analysis."

Acknowledgements Financial support of Russian Foundation for Basic Research (project No. 07-03-96070-r_ural_a) and International Science and Technology Center (projects Nos. 342, 2132 and 2897) is gratefully acknowledged.

Open Access This article is distributed under the terms of the Creative Commons Attribution Noncommercial License which permits any noncommercial use, distribution, and reproduction in any medium, provided the original author(s) and source are credited.

\section{References}

1. Bond AM, Mahon PJ, Schiewe J et al (1997) Anal Chim Acta 345:67

2. Demetriades D, Economou A, Voulgaropoulos A (2004) Anal Chim Acta 519:167

3. Sun J-J, Guo L, Zhang D-F et al (2007) Electrochem Commun 9:283

4. Vakhobova RU, Rakhmonberdyev AD, Rachinskaya GF et al (1993) J Anal Chem 48:1053

5. Hu Z, Seliskar CJ, Heineman WR (1998) Anal Chim Acta 369:93

6. Liu J, Wang X, Chen G et al (2001) Analyst 126:1404

7. Yin Q, Brandon NP, Kelsall GH (2000) J Appl Electrochem 30:1109

8. Kamenev AI, Lushov KA (2001) J Anal Chem 56:429

9. Viter IP, Kamenev AI, Sidakov AA et al (1994) J Anal Chem 49:1295

10. Kamenev AI, Kovalenko MA (2000) J Anal Chem 55:659

11. Nadezhina LS, Pronina OV, Yakovleva NYu (1995) J Anal Chem 50:1164

12. Filipe OMS, Brett CMA (2003) Talanta 61:643

13. Brett CMA, Angnes L, Liess HD (2001) Electroanalysis 13:765

14. Pauliukaite R, Brett CMA (2005) Electroanalysis 17:1354

15. Huang M, Huang MJ, Faguy PW et al (1997) Electroanalysis 9:1201 
16. Gouveia-Caridade C, Pauliukaite R, Brett CMA (2006) Electroanalysis 18:854

17. Liu Z, Huan S, Jiang J et al (2006) Talanta 68:1120

18. Banks CE, Compton RG (2006) Analyst 131:15

19. Wildgoose GG, Banks GE, Leventis HC et al (2006) Microchim Acta 152:187

20. Wantz F, Banks CE, Compton RG (2005) Electroanalysis 17:655

21. Banks CE, Compton RG (2005) Anal Sci 21:1263

22. Kachoosangi RT, Banks CE, Ji X, Compton RG (2007) Anal Sci 23:283

23. Zeng A, Liu E, Tan SN et al (2002) Electroanalysis 14:1110

24. Zeng A, Liu E, Tan SN et al (2002) Electroanalysis 14:1294

25. Kruusma J, Tomcik P, Banks CE et al (2004) Electroanalysis $16: 852$

26. Kruusma J, Banks CE, Lust E et al (2004) Electroanalysis 16:596

27. Babyak C, Smart R (2004) Electroanalysis 16:175

28. Tsai YC, Coles BA, Holt K et al (2001) Electroanalysis 13:831

29. Prado C, Wilkins SJ, Gründler P et al (2003) Electroanalysis 15:1011

30. Goodwin A, Lawrence AL, Banks CE et al (2005) Anal Chim Acta 533:141

31. Banks CE, Hyde ME, Tomčík P et al (2004) Talanta 62:279

32. Sonthalia P, McGaw E, Show Y et al (2004) Anal Chim Acta $522: 35$

33. Kruusma J, Banks CE, Compton RG (2004) Anal Bioanal Chem 379:700

34. Manivannan A, Ramakrishnan L, Seehra MS et al (2005) J Electroanal Chem 577:287

35. Song Y, Swain GM (2007) Anal Chem 79:2412

36. Goeting CH, Jones F, Foord JS et al (1998) J Electroanal Chem 442:207

37. Maeda Y, Ramaraj R, Rao TN et al (1999) Electrochim Acta 44:3441

38. Saterlay AJ, Marken F, Foord JS et al (2000) Talanta 53:403

39. Chatterjee A, Wiltshire R, Holt KB et al (2002) Diamond Relat Mater 11:646

40. McGaw EA, Swain GM (2006) Anal Chim Acta 575:180

41. Manivannan A, Kawasaki R, Tryk DA (2004) Electrochim Acta 49:3313

42. Dragoe D, Spătaru N, Kawasaki R et al (2006) Electrochim Acta $51: 2437$

43. Ghanem MA, Hanson H, Compton RG et al (2007) Talanta $72: 66$

44. Einaga Y, Sato R, Olivia $\mathrm{H}$ et al (2004) Electrochim Acta 49:3989

45. Roustom SEI, Foti G, Comninellis C (2005) Electrochem Commun 7:398

46. Saez V, Gonzalez-Garcia J, Kulandainathan MA et al (2007) Electrochem Commun 9:1127

47. Colley AL, Wiilliams CG, Johansson UD et al (2006) Anal Chem 78:2539

48. Banks CE, Kruusma J, Moore RR et al (2005) Talanta 65:423

49. Tall OE, Jaffrezic-Renault N, Sigaud M et al (2007) Electroanalysis 19:1152

50. Song Y, Swain GM (2007) Anal Chim Acta 593:7

51. Tallman DE, Peterson SL (1990) Electroanalysis 2:499

52. Gun G, Tsionsky M, Lev O (1994) Anal Chim Acta 294:261

53. Rabinovich L, Lev O (2001) Electroanalysis 13:265

54. Chung DDL (2004) J Mater Sci 39:2645

55. Jin Y, Xu F, Miwa T (2000) Electroanalysis 12:610

56. Kirushov VN (2000) Zavodsk Lab 66/6:16

57. Kirushov VN, Vais AA, Masly AI (2000) Zavodsk Lab 66/8:9

58. Kirushov VN, Vais AA, Skvortsova LI (2001) Zavodsk Lab 67/12:16
59. Matakova RN, Kenzhekhanova ZhT, Badavamova GL (1999) J Anal Chem 54:1188

60. Kirushov VN, Kletenik YuB (1997) Zavodsk Lab 63/12:13

61. Bek RYu, Skvortsova LI, Kirushov VN et al (2001) Zavodsk Lab 67/2:3

62. Kletenik YuB, Aleksandrova TP (1997) J Anal Chem 52:280

63. Wang J, Kirgöz UA, Mo JW et al (2001) Electrochem Commun 3:203

64. Zimer AM, Bertholdo R, Grassi MT et al (2003) Electrochem Commun 5:983

65. Stefan RI, Bairu SG (2003) Anal Chem 75:5394

66. Dijk N, Fletcher S, Madden CE (2001) Analyst 126:1878

67. Cordero-Rando MM, Hidalgo-Hidalgo de Cisneros JL, Blanco E et al (2002) Anal Chem 74:2423

68. Opallo M, Saczek-Maj M (2001) Electrochem Commun 3:306

69. O'Hare D, MacPherson JV, Willows A (2002) Electrochem Commun 4:245

70. Carregalo S, Merkoçi A, Alegret S (2004) Microchim Acta 147:245

71. Scvortsova LI, Aleksandrova TP, Tarasova VA et al (2005) J Anal Chem 60:1284

72. Ramirez-Garcia S, Alegret S, Cespedes F et al (2002) Analyst 127:1512

73. Kahlert H, Scholz F (1997) Electroanalysis 9:922

74. Khustenko LA, Larina LN, Nazarov BF (2003) J Anal Chem $58: 297$

75. Khustenko LA, Larina LN (2005) Zavodsk Lab 71/4:9

76. Kopanica M, Stara V (1991) Electroanalysis 3:925

77. Wang J, Pamidi PVA, Nascimento VB et al (1997) Electroanalysis 9:689

78. Khoo SB, Ye R (2002) Electroanalysis 14:420

79. Ji Z, Guadalupe AR (1999) Electroanalysis 11:167

80. Stozhko NYU, Morosanova EI, Kolyadina LI, Fomina SV (2006) J Anal Chem 61:170

81. Zejli H, Sharrock P, Hidalgo-Hidalgo de Cisneros JL et al (2005) Talanta $68: 79$

82. Majidi MR, Asadpour-Zeynali K, Hosseini S-A (2007) Electroanalysis 19:364

83. Wang J, Brennsteiner A, Angnes L (1992) Anal Chem 64:151

84. Khan MR, Khoo SB (2001) Analyst 126:2172

85. Khoo SB, Ye R (1999) Analyst 125:895

86. Navrátil T, Šebková S, Kopanika M (2004) Anal Bioanal Chem 379:294

87. Albertus F, Allerena A, Alpizar J et al (1997) Anal Chim Acta 355:23

88. Tarasova VA, Kletenik YuB (1998) Zavodsk Lab 64/2:9

89. Bek RYu, Aleksandrova TP, Skvortsova LI et al (2002) Electroanalysis 14:1017

90. Seo K, Kim S, Parc J (1998) Anal Chem 70:2936

91. Shpigun LK, Kopytova NE (1997) Zavodsk Lab 63/3:5

92. Shpigun LK, Kopytova NE, Kamilova PM et al (1997) J Anal Chem 52:974

93. Domenech-Carbo A, Domenech-Carbo MT, Gimeno-Adelantado JV et al (2001) Analyst 126:1764

94. Almeida CMVB, Giannetti BF (2002) Electrochem Commun 4:985

95. Labuda J, Vaničková M, Beinrohr E (1989) Microchimica Acta $1: 113$

96. Kolpakova NA, Kaminskaya OV, Yagovkina EV (1998) Zavodsk Lab 64/4:9

97. Kolpakova NA, Smyshlaeva EA, Tuzikova SA et al (2003) J Anal Chem 58:303

98. Tsupko TG, Turian YaI, Temerdashev ZA et al (1993) J Anal Chem 48:1947 
99. Filichkina OG, Zakharova EA, Slepchenko GB (2004) J Anal Chem 59:541

100. Viltchinskaia EA, Zeigman LL (1996) Electroanalysis 8:92

101. Lukaszewski Z, Zembrzuski W, Piela A (1996) Anal Chim Acta 318:159

102. Brainina KZ, Viltchinskaia EA, Khanina RM et al (1992) Electroanalysis 4:549

103. Tur'yan YI, Strochkova EM, Kuselman I (1996) Fresenius J Anal Chem 354:410

104. Zakharova EA, Pichugina VM, Pikula NP (1998) Zavodsk Lab 64/5:9

105. Svintsova LD, Kaplin AA, Vartanian SV (1991) J Anal Chem 46:896

106. Zakharova EA, Filichkina OG, Pikula NP (1998) Zavodsk Lab $65 / 2: 3$

107. Filichkina OG, Zeeman LP, Zakharova EA et al (1999) Zavodsk Lab 65/2:8

108. Zakharova EA, Pichugina VM, Tolmacheva TL (1996) J Anal Chem 51:1000

109. Batalova IR, Zakharova EA, Slepchenko GB et al (2004) J Anal Chem 59:528

110. Kamenev AI, Liakhov AB, Orlov SE (2005) J Anal Chem 60:179

111. Korolczuk M (1997) Fresenius J Anal Chem 357:389

112. Viltchinskaia EA, Zeigman LL, Morton SG (1995) Electroanalysis 7:264

113. Kamenev AI, Orlov SE, Liakhov AB (2001) J Anal Chem 56:962

114. Nadezhina LS, Grilikhes MS, Demin VA et al (1994) J Anal Chem 49:974

115. Labuda J, Vanichkova M, Pavlischuk VV et al (1992) J Anal Chem 47:1297

116. Tekutskaia EE, Kravtsov VI (1998) Zavodsk Lab 65/7:8

117. Tekutskaia EE, Kondrat'ev VV, Osipova MV (1999) J Anal Chem 54:1289

118. Ye R, Khoo SB (1999) Analyst 124:353

119. Ye R, Khoo SB (1997) Electroanalysis 9:481

120. Malakhova NA, Chernysheva AV, Brainina KhZ (1991) Electroanalysis $3: 803$

121. Desimoni E, Genevini P, Tambone F et al (2000) Electroanalysis $12: 337$

122. Brainina KhZ, Chernysheva AV, Nikitina NA (1990) Zavodsk Lab 56/7:17

123. Popkova GN, Fedorova ND, Brainina KhZ (1991) J Anal Chem 46:778

124. Stenina LE, Chernysheva AV, Brainina KhZ (1997) Zavodsk Lab $63 / 5: 1$

125. Tsymbal MV, Turjan IY, Temerdashev ZA et al (1994) Electroanalysis 6:113

126. Brainina KHZ, Tsymbal MV, Temerdashev ZA (1992) Zavodsk Lab 58/3:11

127. Malakhova NA, Popkova GN, Wittman G et al (1996) Electroanalysis 8:375

128. Tobolkina NV, Fedorova ND (1993) Zavodsk Lab 59/12:16

129. Tobolkina NV, Fedorova ND, Brainina KhZ (1992) Zavodsk Lab 58/5:5

130. Grygar T, Marken F, Schröder U, Scholz F (2002) Collect Czech Chem Commun 67:163

131. Walcarius A (2006) J Solid State Electrochem 10:469

132. Scholz F, Nitschke L, Henrion G (1990) Electroanalysis 2:85

133. Scholz F, Lange B (1992) Trends Anal Chem 11:359

134. Zhang S, Meyer B, Moh G, Scholz F (1995) Electroanalysis 7:319

135. Doménech-Carbó A, Moya-Moreno M, Domenech-Carbo MT (2004) Anal Bioanal Chem 380:146

136. Doménech-Carbó A, Sánchez-Ramos S, Yusá-Marco DJ et al (2004) Anal Chim Acta 501:103
137. Fiedler DA, Albering JH, Besenhard JO (1998) J Solid State Electrochem 2:413

138. Ciglenečki I, Bura-Nakic E, Inzelt G (2007) Electroanalysis 19:1437

139. Brainina KhZ, Malakhova NA, Stojko NYu (2000) Fresenius J Anal Chem 368:307

140. Reeder GS, Heineman WR (1998) Sensors and Actuators 52:58

141. Honeychurch KC, Hawkins DM, Hart JP et al (2002) Talanta $57: 565$

142. Honeychurch KC, Hart JP, Cowell DC (2000) Electroanalysis $12: 171$

143. Wang J, Musameh M (1994) Analyst 129:25

144. Mooring L, Karousos NG, Livingstone C et al (2005) Sensors and Actuators B: Chemical 107:491

145. Bagel O, Lagger G, Girauult HH et al (2001) Electroanalysis 13:100

146. Renedo O, Martinez JA (2007) Electrochem Commun 9:820

147. Renedo O, Martinez JA (2007) Anal Chim Acta 589:255

148. Wang J, Tian B (1993) Anal Chim Acta 274:1

149. Wang J, Lu J, Hocevar S et al (2001) Electroanalysis 13:13

150. Liu TZ, Lai D, Osterioh JD (1997) Anal Chem 69:3539

151. Wang J, Tian B (1992) Anal Chem 64:1706

152. Desmond D, Lane B, Alderman J et al (1998) Sens Actuators B Chem 48:409

153. Jasinski M, Grundler P, Flechsig GU et al (2001) Electroanalysis $13: 34$

154. Hart JP, Wring SA (1996) Electroanalysis 8:617

155. Wang J, Lu J, Tian B et al (1993) J Electroanal Chem 361:77

156. Zen JM, Chung HH, Kumar AS (2000) Anal Chim Acta 421:189

157. Brainina Kh, Schafer H, Ivanova A et al (1996) Anal Chim Acta $330: 175$

158. Brainina KhZ, Bond AM (1995) Anal Chem 67:2586

159. Weston MC, Anderson EC, Arumugan PU et al (2006) Analyst 131:1322

160. Wang J, Rongrong X, Baomin T et al (1994) Anal Chim Acta 293:43

161. Palchetti I, Cagnini A, Mascini M, Turner APF (1999) Microchim Acta 131:65

162. Palchetti I, Laschi S, Mascini M (2005) Anal Chim Acta 530:61

163. Choi JY, Seo K, Cho SR et al (2001) Anal Chim Acta 443:241

164. Malakhova NA, Miroshnikova EG, Stojko NYu, Brainina KhZ (2004) Anal Chim Acta 516:49

165. Stozhko NYu, Kozitsina AN, Chiavarini S et al (2007) Ital J Food Sci 19:254

166. Stojko NYu, Inzhevatova OV, Koljadina LI (2005) J Anal Chem 60:747

167. Malakhova NA, Stojko NYu, Brainina KhZ (2007) Electrochem Commun 9:221

168. Brainina Kh, Malakhova N, Ivanova A (1998) Solid carbon containing stripping voltammetry sensors. In: Nikolelis DP (ed) Biosensors for direct monitoring of environmental pollutants in field. Kluwer Academic, Norwell, pp 255-269

169. Brainina Kh, Henze G, Stojko N et al (1999) Fresenius J Anal Chem 364:285

170. Brainina KhZ, Ivanova AV, Malakhova NA (1997) Anal Chim Acta 349:85

171. Faller C, Henze G, Stojko N et al (1997) Fresenius J Anal Chem 358:670

172. Stojko N, Brainina Kh, Faller C, Henze G (1998) Anal Chim Acta $371: 145$

173. Faller C, Stojko N, Henze G, Brainina Kh (1999) Anal Chim Acta 396:195

174. Brainina KhZ, Stojko NYu, Shalygina ZhV (2002) J Anal Chem $57: 1116$ 
175. Stojko NYu, Inzhevatova OV (2004) J Anal Chem 59:949

176. Stojko NYu, Lipunova GN, Maslakova TI et al (2004) J Anal Chem 59:202

177. Stojko NYu, Inzhevatova OV, Koljadina LI, Lipunova GN (2005) J Anal Chem 60:187

178. Wang J, Nascimento VB, Lu J et al (1996) Electroanalysis 8:635

179. Stojko NYu, Morosanova EI, Koljadina LI et al (2006) J Anal Chem 61:170

180. Akhtar P, Devereaux HA, Downard AJ et al (1999) Anal Chim Acta 381:49

181. Stojko NYu, Morosanova EI, Koljadina LI, Azarova ZhM (2004) J Anal Chem 59:960

182. Stojko NYu, Morosanova EI, Koljadina LI, Fomina SV (2005) Zavodsk Lab 71/3:14

183. Yantasee W, Deibler LA, Flyxell GE et al (2005) Electrochem Commun 7:1170

184. Yantasee W, Flyxell GE, Lin Y (2006) Analyst 131:1342

185. Honeychurch KC, Hart JP, Cowell DC et al (2001) Sensors and Actuators B Chemical 77:642

186. Honeychurch KC, Hart JP, Cowell DC et al (2002) Electroanalysis $14: 177$

187. Parat C, Betelu S, Authier L et al (2006) Anal Chim Acta 573$574: 14$

188. Honeychurch KC, Hart JP, Cowell DC (2001) Anal Chim Acta 431:89

189. Shin Y, Kumar AS, Zen JM et al (2005) Bull Jpn Chem Soc 78:2130

190. Ugo P, Moretto LM, Bertoncello P et al (1998) Electroanalysis 10:1017

191. Neuhold CG, Wang J, Nascimento VB et al (1995) Talanta 42:1791

192. Wang J, Pedrero M, Sakslund H et al (1996) Analyst 121:345

193. Maystrenko VN, Rusakov IA, Budnikov GK (1992) J Anal Chem 47:1003

194. Štulik K (1999) Electroanalysis 11:1001

195. Feeney R, Kounaves SP (2000) Electroanalysis 12:677

196. Zoski CG (2002) Electroanalysis 14:1041

197. Budnikov GK, Kazakov VE, Poliakov YuN et al (1994) J Anal Chem 49:410

198. Matysik FM, Gläser P, Werner G (1994) Fresenius J Anal Chem 349:646

199. Baranski AS (2002) Anal Chem 74:1294

200. Doronin AN, Muntianu GG (1984) J Anal Chem 39:607

201. Zhao G, Giolando DM, Kirchhoff JR (1995) Anal Chem 67:2592
202. Mylonakis A, Economou A, Fielden PR et al (2004) Electroanalysis $16: 524$

203. Kamenev AI, Sidorov NV (1997) J Anal Chem 52:746

204. Muntianu GG (1998) J Anal Chem 53:1070

205. Muntianu GG (1998) J Anal Chem 53:312

206. Silva SM (1998) Electroanalysis $10: 722$

207. Silva SM, Bond AM (2003) Anal Chim Acta 500:307

208. Baldo MA, Daniele S, Mazzocchin GA (1998) Electroanalysis $10: 410$

209. Muntianu GG (2000) J Anal Chem 55:979

210. Sanna G, Pilo MI, Piu PC et al (2002) Electroanalysis $14: 1512$

211. Spano N, Panzanelli AA, Piu PC et al (2005) Anal Chim Acta 553:201

212. Bartlett PN, Denuault G, Sousa MFB (2000) Analyst 125:1135

213. Wojciechowski M, Balcerzak J (1991) Anal Chim Acta 249:433

214. Bumber AA, Nechitaylov YuN, Profatilova IA et al (2000) J Anal Chem 55:742

215. Barbosa RM, Rosario LM, Brett CMA et al (1996) Analyst 121:1789

216. Daniele S, Bragato C, Baldo MA (1997) Anal Chim Acta 346:145

217. Muntianu GG (2004) J Anal Chem 59:851

218. Baldo MA, Daniele S, Mazzocchin GA (1998) Electroanalysis 10:410

219. Petrovic SC, Dewald HD (1997) Anal Chim Acta 357:33

220. Emons H, Baade A, Schoning MJ (2000) Electroanalysis $12: 1171$

221. Muntianu GG (2001) J Anal Chem 56:614

222. Wang J, Lu J, Hocevar SB, Farias PAM (2000) Anal Chem $72: 3218$

223. Lin L, Thongngamdee S, Wang J et al (2005) Anal Chim Acta 535:9

224. Hutton EA, Hočevar SB, Ogorevc B (2005) Anal Chim Acta 537:285

225. Wang J, Armalis S (1995) Electroanalysis 7:958

226. Schiewe J, Oldham KB, Myland JC et al (1997) Anal Chem 69:2673

227. Feldman BJ, Osterioh JD, Hata BH et al (1994) Anal Chem 66:1983

228. Liu G, Lin Y, Tu Yi et al (2005) Analyst 130:1098

229. Wang J, Tian B, Wang J et al (1999) Anal Chim Acta 385:429

230. Hernandez-Santos D, Gonzalez-Garcia MB, Garcia AC (2002) Electroanalysis 14:1225

231. Katz E, Willner I, Wang J (2004) Electroanalysis 16:19

232. Welch CM, Compton RG (2006) Anal Bional Chem 384:601 